ARTICLE

\title{
Implementation of Multileaf Collimator in a LINAC MCNP5 Simulation Coupled with the Radiation Treatment Planing System PLUNC
}

\author{
Vicente ABELLA, Rafael MIRÓ, Belén JUSTE and Gumersindo VERDÚ* \\ Institute for Industrial, Radiophysical and Environmental Safety (ISIRYM), \\ Universitat Politècnica de València, \\ Camí de Vera s/n, 46021 València, Spain
}

\begin{abstract}
Multileaf collimators are used on linear accelerators to provide conformal shaping of radiotherapy treatment beams, being an important tool for radiation therapy dose delivery. In this work, a multileaf collimator has been designed and implemented in the MCNP model of an Elekta Precise Linear Accelerator (LINAC) and introduced in PLUNC, a set of software tools for radiotherapy treatment planning (RTP) which was coupled in previous works with MCNP5 (Monte Carlo N-Particle transport code), with the purpose of comparing its effect on deterministic and Monte Carlo dose calculations. A 3D Shepp-Logan phantom was utilized as the patient model for validation purposes.

Once the multileaf collimator model is implemented in the PLUNC LINAC model, a series of Matlab interfaces extract phantom and beam information created with PLUNC during the treatment plan and write it in MCNP5 input deck format. After the Monte Carlo simulation is performed, results are input back again in PLUNC in order to continue with the plan evaluation.

The comparison is made via mapping of dose distribution inside the phantom with different field sizes, utilizing the MCNP5 tool FMESH, superimposed mesh tally, which allows registering the results over the problem geometry. This work follows a valid methodology for multileaf LINAC MC calculations during radiation treatment plans.
\end{abstract}

KEYWORDS: Monte Carlo, PLUNC, radiotherapy, treatment plan, Shepp-Logan phantom

\section{Introduction}

Monte Carlo simulation techniques are nowadays becoming an important tool for patient dose calculation in radiotherapy treatment planning systems. It has been shown that $\mathrm{MC}$ techniques provide a more accurate dose calculation compared to 'conventional' treatment planning systems, with the only limitation of computing time $\operatorname{cost}^{1)}$.

Multileaf collimators (MLC) are used on linear accelerators to provide conformal shaping of radiotherapy treatment beams. It is an important tool for radiation therapy dose delivery. In this work, a MLC has been designed and implemented in the MCNP model of an Elekta Precise Linear Accelerator and introduced in PLUNC, ${ }^{2)}$ a set of software tools for radiotherapy treatment planning (RTP) which was coupled in previous works with MCNP5 (Monte Carlo N-Particle transport code), ${ }^{3)}$ with the purpose of comparing its effect on deterministic and Monte Carlo dose calculations. MCNP5 code uses photoneutrons in transport calculations and therefore neutrons created by photons can be registered whereas other Monte Carlo codes such as PENELOPE, EGS4 or DPM do not have this feature.

In the RTP context, the reconstruction of a mathematical phantom is a very popular way to validate dose calculation algorithms. Following this, the Shepp-Logan phantom is widely regarded as a gold standard for that purpose. ${ }^{4)}$ The Shepp-Logan phantom consists of ten ellipsoids with differ-

*Corresponding author, E-mail: gverdu@iqn.upv.es ent pixel intensities. The phantom is provided inside Matlab code and is converted into dicom format in order to input the image slices into the planning system. In the end, a 3D Shepp-Logan phantom is utilized as the patient model in order to compare calculations provided by PLUNC with those obtained via MCNP5 simulation of the LINAC irradiation with the multileaf collimator.

\section{Materials and Methods}

\section{Description of the MLC Model and the Coupling Me- thod}

An MCNP5 Elekta Precise MLC model was previously validated $^{5,6)}$ at $6 \mathrm{MeV}$. The model includes the major components of the MLC accelerator head. Dose calculations with this model involve collimated beams by a large number of small. Since such calculations are very sensitive to the detailed structure of the multileaf collimator, the 80-leaf MLC Elekta Precise was implemented in a geometric developed model. The simulation of the validated model with different field sizes provides a series of phase-space files which store the particle information so that, in future simulations, these source files can be used by changing the position, when necessary, according to the gantry, table and collimator angle, significantly reducing the computing time.

To achieve accurate dose computation in PLUNC for a treatment unit, which refers to a physical machine operating at a set energy level and modality, its detailed physical and 
dosimetric descriptions is established through the PLUNC commissioning process, by which the Elekta Precise Linear Accelerator was implemented in PLUNC. This way, PLUNC plan can be performed with the same MLC LINAC model that was validated for the MCNP5 simulations and the Elekta machine appears as an option for the beam settings after the commissioning process.

The spectrum introduced in PLUNC has been obtained after the simulation of the acceleration of a monodirectional electron point source beam upon the tungsten target using a $6.3 \mathrm{MeV}$ initial electron energy with a radial gaussian FWHM spatial distribution of 0.11 (data supplied by Elekta). Figure 1 shows the photon spetrum used.

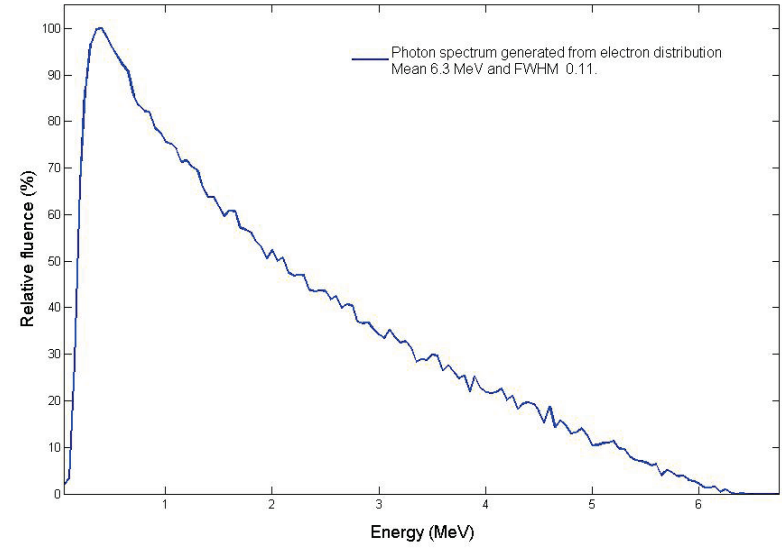

Fig. 1 Photon spectrum generated after an electron energy dis-tribution with mean $=6.3 \mathrm{MeV}$ and $\mathrm{FWHM}=0.11$

MCNP5 has been coupled with PLUNC via a series of Matlab interfaces, so the simulation of the irradiation of the patient with different field sizes provides the radiophysicist with an alternative accurate Monte Carlo simulation. PLUNC software includes a full range of RTP functions including image importing and processing, virtual simulation, dose calculation, plan evaluation, and planning for intensity modulated radiotherapy.

Patient and beam information created with PLUNC can be translated to MCNP5 code and the simulation performed with the desired field size. Once the Monte Carlo simulation is finished, results are input back again in PLUNC in order to evaluate the treatment plan.

\section{Shepp-Logan Phantom}

The Shepp-Logan phantom is a well known standard for dose algorithm validation purposes. This numerical phantom consists of ten ellipsoids with different pixel intensities. The phantom is provided inside Matlab code, which allows the user to set the size of the ellipsoids, the pixel intensities and the number of pixels in $x, y$ and $z$. The program is designed to convert the numerical phantom into dicom format, so the image slices can be input into the planning system.

An inside cut of the Shepp-Logan numerical phantom, sliced in the $z$ axis can be seen in Fig. 2.

The Shepp-Logan numerical phantom was designed in this work to have 9 ellipsoids with 3 different pixel intensi-

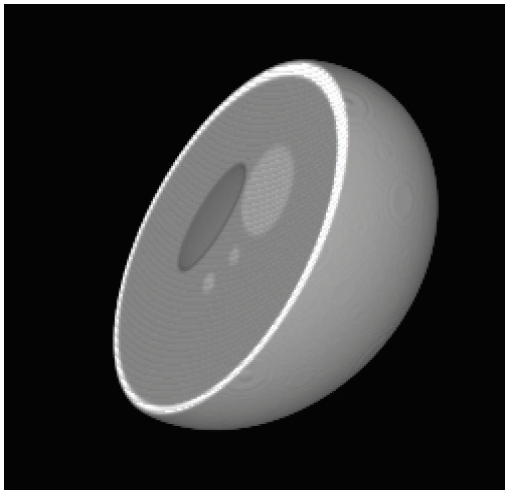

Fig. 2 Inside cut of the Shepp-Logan numerical phantom

ties, so three materials would simulate the basic body composition: the soft tissue material $(0.997 \mathrm{~g} / \mathrm{cc})$, the skeleton $(1.61 \mathrm{~g} / \mathrm{cc})$ and the air $(0.0013 \mathrm{~g} / \mathrm{cc})$.

A set of 128 dicom files simulating Computer Tomography slices was obtained with an image resolution of $128 \times$ 128 pixels and 16 bits per pixel, separated by $0.2 \mathrm{~cm}$ one from the other.

This set of images was input in PLUNC, by converting the 128 dicom images into PLUNC's single image file plan_im, and subsequently the segmentation of the images was performed via the PLUNC anastruct editor, obtaining two different anatomical structures, the soft tissue and the skeleton. The Matlab interfaces created by the authors read the phantom information via PLUNC MC Interface and write it in the MCNP5 input deck format, taking into account the size of the segmented phantom and the position where the beam is focused to give $100 \%$ of the dose. The MC Interface reinterprets the phantom and assigns a number of voxels according to the isocenter positioning necessities, resulting in this case an $84 \times 112 \times 98$ voxels phantom. The MCNP5 lattice card is used to depict de voxel geometry, reducing this way the computing time around 6 times. ${ }^{7)}$

The three-dimensional voxelized phantom MCNP5 model can be seen in Fig. 3, visualized with Sabrina code. The final model of the Shepp-Logan phantom is a 921,984 voxels lattice structure.

PLUNC performs the dose calculation utilizing the Clarkson Integrated Batho-homogeneity-corrected algorithm,

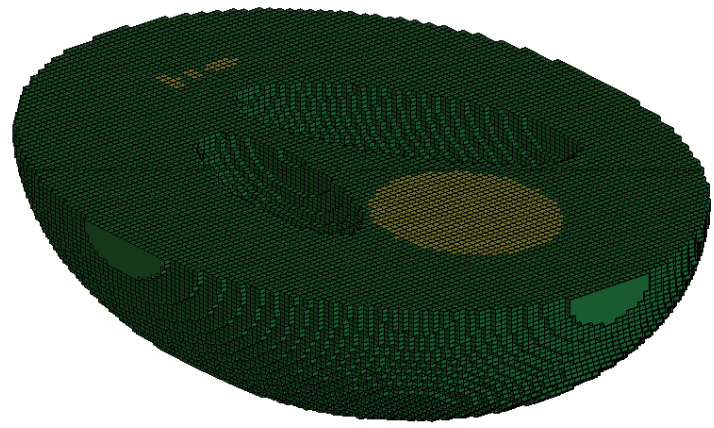

Fig. 3 Voxelization of the Shepp-Logan phantom, inside cut, visualization by Sabrina code 
and in this case assuming the densities fixed by the segmentation process instead of the Hounsfield numbers. The dose grid obtained in the simulation with MCNP5 is input back in PLUNC.

\section{MCNP Simulation}

The results of the Monte Carlo simulation depend on the strictness of the Monte Carlo simulation model. Several parameters need to be taken into account, as the physics, material properties, geometry specifications, source characteristics, variance reduction techniques, detector tallies and the set of the number of particles to track.

A detailed photon physics treatment, including photoelectric effect with fluorescence production, incoherent and coherent scattering and pair production, has been considered in the energy range between 0.001 and $7 \mathrm{MeV}$. The photon energy cut-off considered for this study used the default value in MCNP, $1 \mathrm{keV}$, while it was set to $100 \mathrm{keV}$ for electrons. An importance ratio of 4 is set in the voxels inside the phantom, in order to reduce the statistical error where most collisions occur.

In the end, we obtain the dose distribution maps inside the phantom, via the FMESH tally, which allows defining a mesh tally superimposed over the problem geometry. Adding the conversion factors for photons and electrons, this feature calculates the dose averaged over a mesh cell, which in our case corresponds to each phantom voxel. Results can be input back in PLUNC and compared with those of relative dose calculated with PLUNC algorithms.

MCNP code has been parallelized in an HP Proliant DL 580, utilizing the MPI parallel protocol, using 16 processors for our simulation. Furthermore, MCNP code has been modified in order to allow geometries up to 2,900,000 lattice voxels ${ }^{8)}$ with the Intel Fortran Compiler 11.1, on the Linux parallel computing machine. The simulation was carried with MCNP5 version 1.40, for 4,058,897,000 initial particles obtaining an average statistical dispersion of around 5\%. For the $12 \mathrm{~cm} \times 12 \mathrm{~cm}$ field simulation, the final simulation real CPU time was 198 minutes.

\section{Results and Discussion}

The validation has been performed by comparing the results of the irradiation of the numerical phantom with different field sizes $(5 \mathrm{~cm} \times 5 \mathrm{~cm}, 12 \mathrm{~cm} \times 12 \mathrm{~cm}$ and $30 \mathrm{~cm}$ $\times 30 \mathrm{~cm})$.

The first two figures present MCNP and PLUNC relative dose map calculations, visualized by PLUNC RTP tools, for the $12 \mathrm{~cm} \times 12 \mathrm{~cm}$ beam. The irradiation was set to give a $100 \%$ of the dose at $100 \mathrm{~cm}$ from the source. All dose values are normalized so that the $100 \%$ of the dosed is delivered at such isocenter. In the MCNP simulation, the beam is centered and focused to the axes origin, coinciding with the isocenter in PLUNC's coordinates, along with the positive $x$ axis.

Figure 4 shows the relative dose distribution calculated by PLUNC, with the homogeneity correction set by taking into account the anastructures density.

Figure 5 shows the relative dose distribution calculated

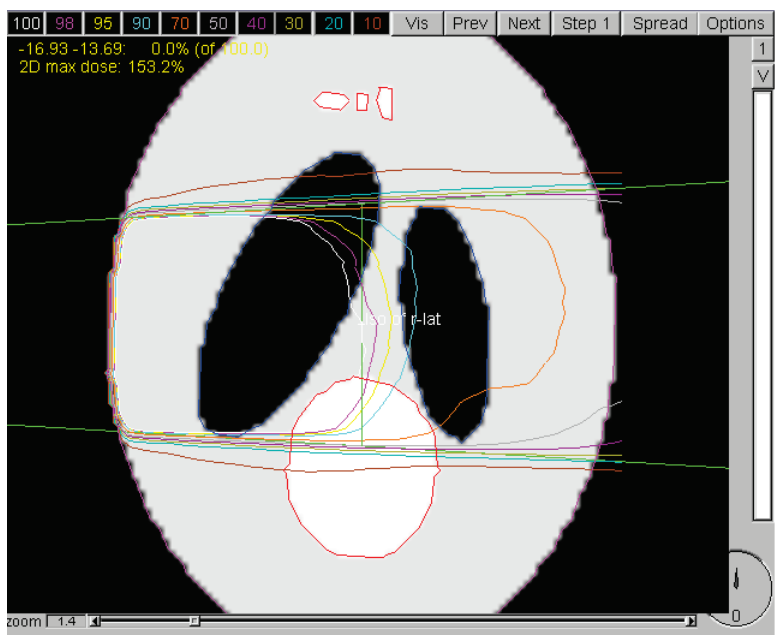

Fig. 4 Relative dose distribution calculated with PLUNC, for a $12 \mathrm{~cm} \times 12 \mathrm{~cm}$ beam

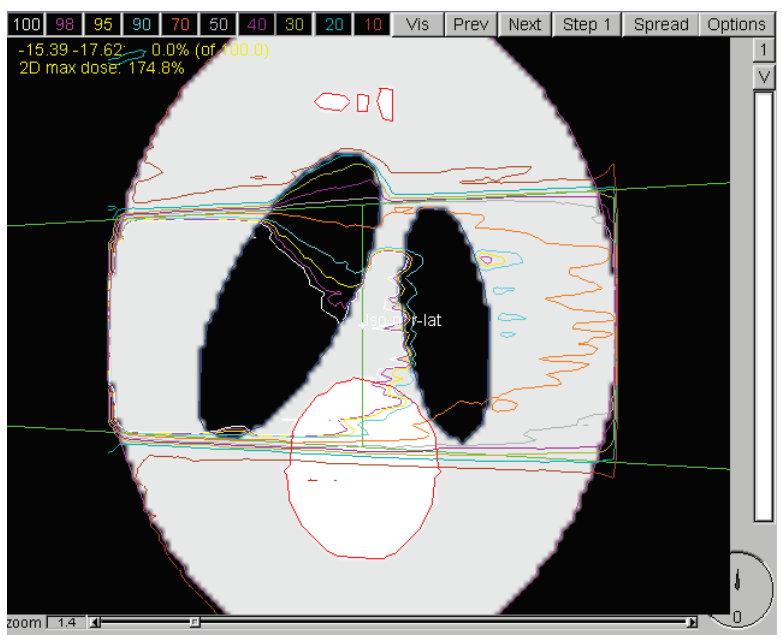

Fig. 5 Relative dose distribution calculated with MCNP5, for a $12 \mathrm{~cm} \times 12 \mathrm{~cm}$ beam

with MCNP5 and returned to PLUNC graphical interface for comparison purposes. This way the plan evaluation can also continue.

It is observed that both relative dose distributions follow a very similar pattern, though MCNP calculations offer a more precise distribution. It is clear that MCNP5 takes into account the dose absorption where a change of material density appears, like from skull to skin or from skin to air.

Figures 6, 7 and 8 present the numerical MCNP5 and PLUNC comparison of the relative dose against the skin structure volume, for the three beams.

Slight differences can be observed in the relative dose against volume graphs.

\section{Conclusion}

This work offers a valid methodology for precise MLC irradiation simulations during radiation treatment plans. It has been shown that MLC can be used in Monte Carlo calculations for RTPs, in specific for PLUNC. More precise 


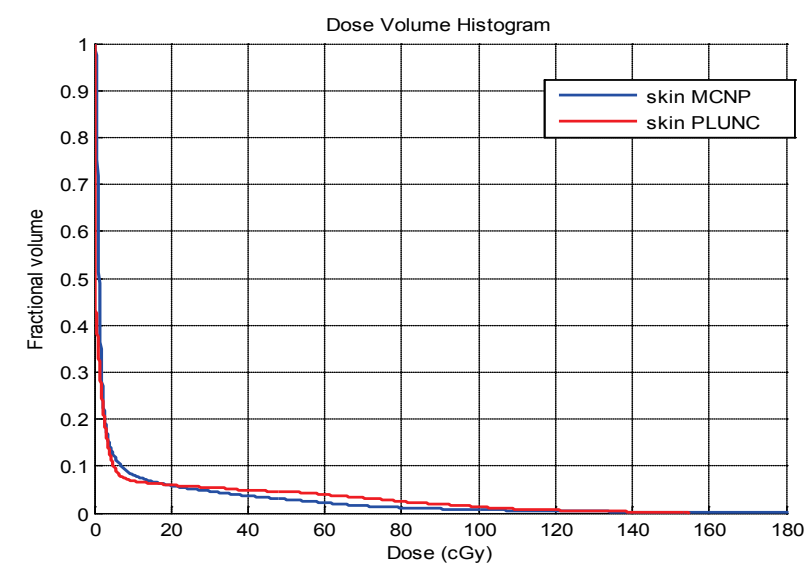

Fig. 6 Dose against skin volume comparison, $5 \mathrm{~cm} \times 5 \mathrm{~cm}$ beam

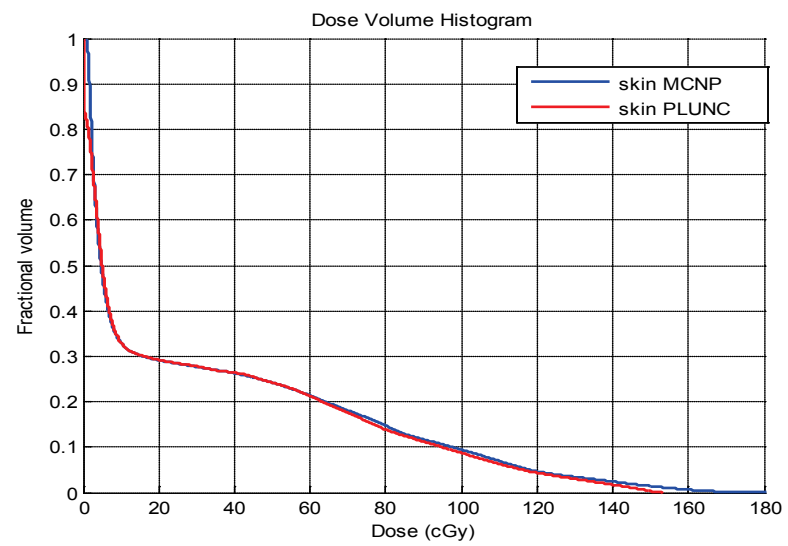

Fig. 7 Dose against skin volume comparison, $12 \mathrm{~cm} \times 12 \mathrm{~cm}$ beam

dose distributions can be obtained with Monte Carlo simulations.

Future works will focus on MLC irradiations with irregular fields as regular fields have been proved to be successful.

\section{References}

1) I. J. Chetty, B. Curran, J. E. Cygler, J. J. DeMarco, G. Ezzell, B. A. Faddegon, I. Kawrakow, P. J. Keall, H. Liu, C. M. C. Ma, D. W. O. Rogers, J. Seuntjens, D. Bagheri, J. V. Siebers, "Report of the AAPM task Group No. 105: Issues associated with clinical implementation of Monte Carlo-based photon and electron external beam treatment planning," Med. Phys., 34[12], 4818-4853 (2007).

2) Department of Radiation Oncology at the University of North Carolina at Chapel Hill, http://planunc.radonc.unc.edu/

3) X-5 Monte Carlo Team, "MCNP- A General Monte Carlo

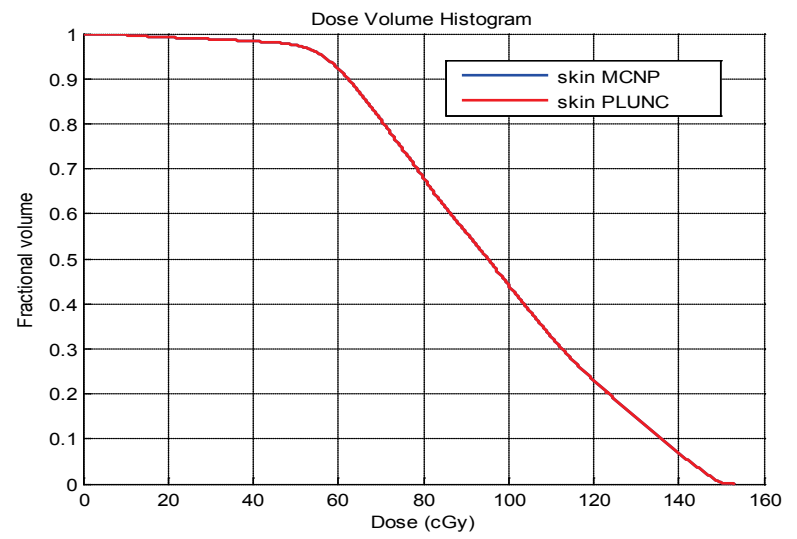

Fig. 8 Dose against skin volume comparison, $30 \mathrm{~cm} \times 30 \mathrm{~cm}$ beam

N-Particle Transport Code Version 5," LA-UR-03-1987, Los Alamos National Labotratory (LANL) (2003).

4) L. A. Shepp, B. F. Logan, "The Fourier reconstruction of a head section," IEEE Trans. Nucl. Sci., 21, 21-34 (1974).

5) B. Juste, A. Husyar, R. Miró, G. Verdú, S. Díeaz, J. M. Campayo, "Experimental validation of an accurate multileaf collimation (MLC) LINAC simulated by Monte Carlo," 6th Workshop on European Collaboration for Higher Education and Research in Nuclear Engineering \& Radiological Protection, Coimbra, Portugal, June 7-9 (2010).

6) V. Abella, R. Miró, B. Juste, G. Verdú, "MCNP5 simulation of the irradiation of a voxelized phantom with the photon beam generated in a Linear Accelerator with a multileaf collimator," 6th Workshop on European Collaboration for Higher Education and Research in Nuclear Engineering \& Radiological Protection, Coimbra, Portugal, June 7-9 (2010).

7) V. Abella, R. Miró, B. Juste, G. Verdú, "Comparison of two differet Methods for describing the Geometry of a Voxelized Anthropomorphic Phantom in Radiotherapy Treatment Plans," Medical Physics and Biomedical Engineering World Congress 2009, Munich, Germany, September 7-12, (2009).

8) V. Abella, R. Miro, B. Juste, A. Santos, G. Verdu, "Monte Carlo model of the female RANDO phantom irradiation with an Elekta Precise linear accelerator," Nucl. Instr. Meth. Phys. Res., A619, 230-233 (2010).

9) H. F. Batho, "Lung corrections in Cobalt 60 beam therapy," $J$. Can. Assoc. Radiol., 15, 39-83 (1964).

10) J. W. Wong, J. A. Purdy, "On methods of inhomogeneity corrections for photon transport," Med. Phys., 17, 807-813 (1990).

11) S. Stathakis, C. Kappas, K. Theodorou, N. Papanikolaou., J. Rosenwald, "An inhomogeneity correction algorithm for irregular fields of high energy photon beams based on Clarkson integration and the 3D Beam Subtraction Method," J. Appl. Clin. Med. Phys., 7[1], 1-13 (2006). 